\title{
Blue irregular variable stars in the Small Magellanic Cloud from EROS2: Herbig Ae/Be or classical Be stars?^
}

\author{
J.-P. Beaulieu ${ }^{1}$, W. J. de Wit ${ }^{1,2,3}$, H. J. G. L. M. Lamers ${ }^{2,3}$, J.-B. Marquette ${ }^{1}$, C. Coutures ${ }^{4}$, P. Leisy ${ }^{5}$,
} S. Totor ${ }^{1}$, N. Palanque-Delabrouille ${ }^{4}$, C. Afonso ${ }^{4}$, C. Alard ${ }^{1}$, J. N. Albert ${ }^{6}$, J. Andersen ${ }^{8}$, R. Ansari ${ }^{6}$,

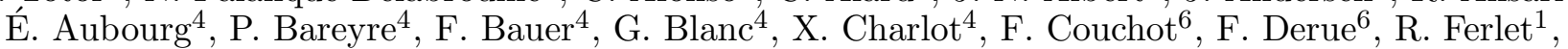
P. Fouqué ${ }^{5,10}$ J. F. Glicenstein ${ }^{4}$, B. Goldman ${ }^{4}$, D. Graff ${ }^{9}$, M. Gros ${ }^{4}$, J. Haissinski ${ }^{6}$, J. C. Hamilton ${ }^{11}$,

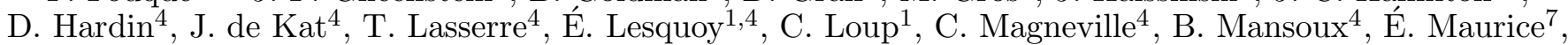

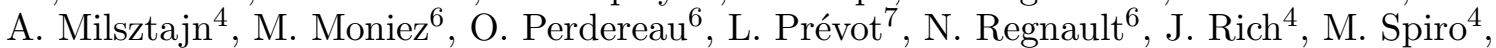
A. Vidal-Madjar ${ }^{1}$, L. Vigroux ${ }^{4}$, and S. Zylberajch ${ }^{4}$

1 Institut d'Astrophysique de Paris, 98bis boulevard Arago, 75014 Paris, France

2 Astronomical Institute, University of Utrecht, Princetonplein 5, 3584 CC, Utrecht, The Netherlands

3 SRON Laboratory for Space Research, Sorbonnelaan 2, 3584 CA, Utrecht, The Netherlands

${ }^{4}$ CEA, DSM, DAPNIA, Centre d'Études de Saclay, 91191 Gif-sur-Yvette, Cedex, France

5 ESO La Silla, casilla 19001, Santiago 19, Chile

${ }^{6}$ Laboratoire de l'Accélérateur Linéaire, IN2P3 CNRS, Université de Paris-Sud, 91405 Orsay Cedex, France

7 Observatoire de Marseille, 2 Pl. Le Verrier, 13248 Marseille Cedex 04, France

8 Astronomical Observatory, Copenhagen University, Juliane Maries Vej 30, 2100 Copenhagen, Denmark

9 Departments of Astronomy and Physics, Ohio State University, Columbus, OH 43210, USA

10 DESPA, Observatoire de Paris section Meudon, 1 place Jules Janssen, Meudon Cedex 92195, France

11 Collège de France, 11 place Marcelin-Bertherlot, 75231 Paris Cedex 05, France

Received 2 May 2001 / Accepted 14 September 2001

\begin{abstract}
Using data from the EROS2 microlensing survey, we report the discovery of two blue objects with irregular photometric behaviour of $\Delta V \sim 0.1-0.4 \mathrm{mag}$ on time scales of 20 to 200 days. They show a bluer when fainter behaviour. Subsequent spectra taken with the ESO $3.6 \mathrm{~m}$ telescope show spectral type B4eIII and B2eIV-V with strong $\mathrm{H} \alpha$ emission. These objects resemble the Herbig AeBe but also classical Be stars. At this stage, it is not possible to distinguish unambiguously between pre-main sequence and classical Be nature. If we favour the pre-main sequence interpretation, they are more luminous than the luminosity upper limit for Galactic HAeBe stars. The same was found for the HAeBe candidates in the LMC. This might be due to a shorter accretion time scale $\left(\tau=M_{*} / \dot{M}\right)$, or the smaller dust content during the pre-main sequence evolution of SMC and LMC stars. Further studies on a larger scale of the environment and IR properties of the stars are needed.
\end{abstract}

Key words. stars - emission line, Be - stars: pre-main sequence - stars: variable: general - gravitational lensing - galaxies: individual: SMC

\section{Introduction}

We present the discovery and the study of two Small Magellanic Cloud blue irregular variable stars. We noticed that during EROS-2 SMC data base mining for microlensing by Palanque-Delabrouille et al. (1998) 10 irregularly blue variable objects were detected (see their Sect. 3 for the method). Among them, two have the same variability characteristics as the Galactic Herbig Ae/Be stars (Herbig 1960; Bibo \& Thé 1991; Waters \& Waelkens 1998 and

\footnotetext{
Send offprint requests to: J. P. Beaulieu,

e-mail: beaulieu@iap.fr

* This work is based on observations at the European Southern Observatory, La Silla, Chile.
}

references therein), and were chosen for deeper analysis, similar to our previous studies of HAeBe candidates in the LMC (Beaulieu et al. 1996; and Lamers et al. 1999; de Wit et al. 2001a, BL, LBD, DBL hereafter). We discuss their photometric and spectroscopic properties, and we present several arguments which might lead to the interpretation of these stars as pre-main sequence (PMS) HAeBe stars. If confirmed, these stars would be the first SMC pre-main sequence stars. Following the nomenclature introduced in BL, we will call the stars EROS SMC HAeBe Candidates or ESHC stars.

A large-scale systematic search over the full EROS2 SMC database as done in the LMC by 

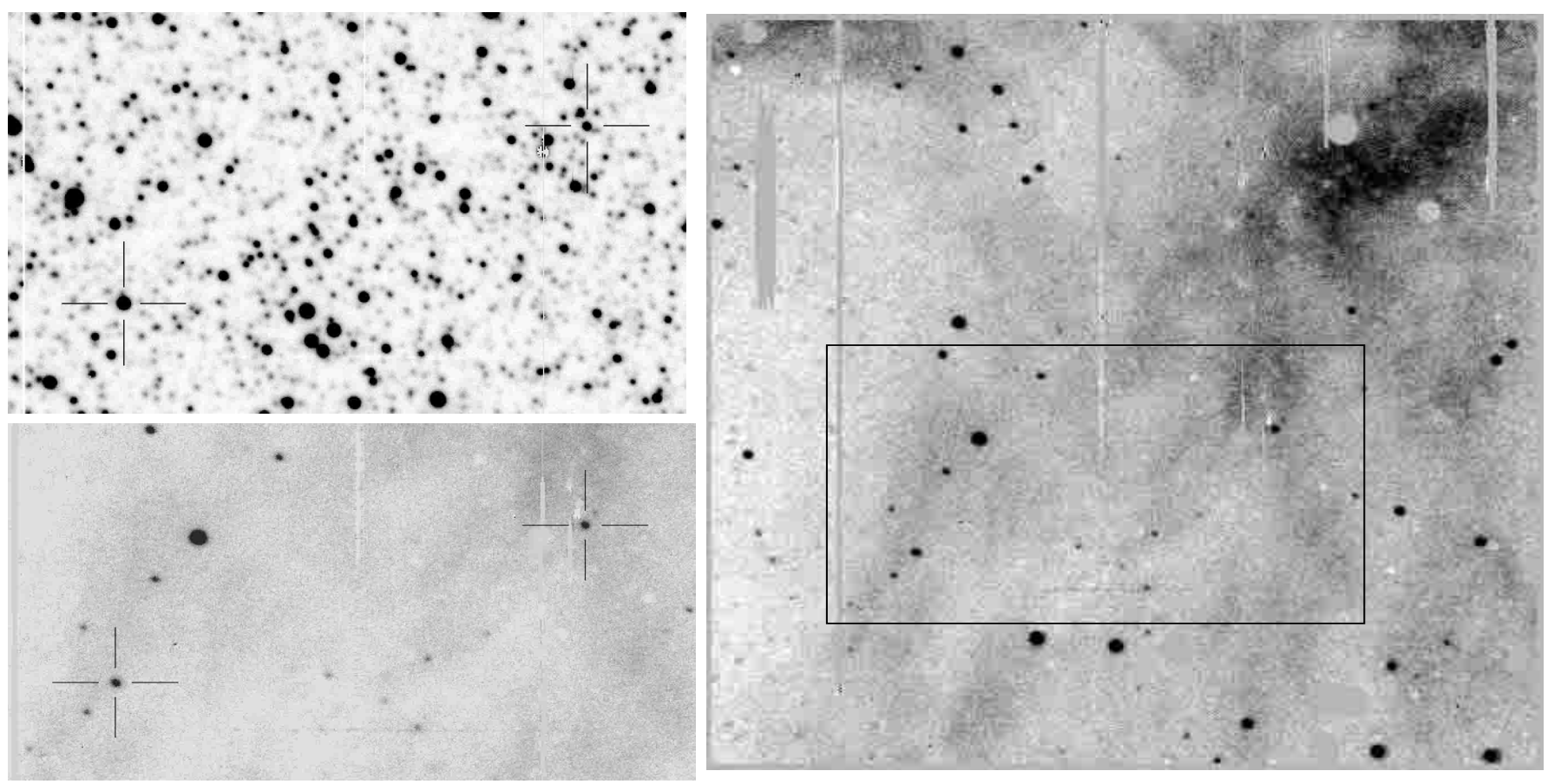

Fig. 1. The upper left panel is the finding chart $(3.25 \operatorname{arcmin} \times 1.65 \operatorname{arcmin})$ in the $\mathrm{H} \alpha_{\text {off }}$ band of the two targets ESHC1 and ESHC2, labeled by the left and the right cross respectively. North is up, East is left. The two other are $(\mathrm{H} \alpha-\mathrm{H} \alpha$ off $) / \sqrt{\mathrm{H} \alpha}$ images. These figures show only the $\mathrm{H}_{\alpha}$ point sources and the $\mathrm{H}_{\alpha}$ emitting regions (stripes are related to data reduction problems like bad columns or saturated stars). The lower left panel is the area shown on the finding chart, the right panel gives the full field observed. One can notice the presence of an H II region, and clusters of $\mathrm{H} \alpha$ emitters.

de Wit et al. (2001a) will be presented in the near future (de Wit et al. 2001b).

\section{The observations}

\subsection{Searching for blue irregular objects in the SMC}

The field has been observed as part of the ongoing EROS2 microlensing survey. The set-up consists of a $1 \mathrm{~m} \mathrm{~F} / 5$ Ritchey-Chrétien telescope with two $2 \times 4$ CCD detectors in different focal planes covering a field of $0.7 \times 1.4$ degree. The light is split by a dichroic allowing simultaneous observing in two non-standard bandpasses $\left(V_{E}=420\right.$ $720 \mathrm{~nm}$ and $R_{E}=620-920 \mathrm{~nm}$ ).

During the search for microlensing in the EROS2 SMC data base (5.3 million stars coming from 10 square degrees of the densest part of the SMC) of one year observations (Palanque-Delabrouille et al. 1998), 10 blue irregular variable objects were discovered which are located in the HRD to the right of the main sequence. Two of these displayed irregular photometric variability with different time scales, superimposed on long term variability. The light curves resemble those of the $\mathrm{HAeBe}$ stars.

The stars, called ESHC1 and ESHC2, have the following coordinates:

ESHC1: $\mathrm{RA}=00: 53: 03.0$ and $\mathrm{DEC}=-73: 17: 59, \mathrm{~J} 2000$

ESHC2: $\mathrm{RA}=00: 52: 32.6$ and $\mathrm{DEC}=-73: 17: 08, \mathrm{~J} 2000$.

The findings charts in $V$ are given in Fig. 1.

The full $V_{\mathrm{E}}$ and $V_{\mathrm{E}}-R_{\mathrm{E}}$ light curves of theses two stars are shown in the two upper panels of Fig. 2. The two lower panels show the low amplitude very irregular photometric behaviour in $V_{\mathrm{E}}$ and $R_{\mathrm{E}}$ for both stars. The uncertainty in the photometry is typically $\sigma V_{\mathrm{E}} \approx \sigma R_{\mathrm{E}} \approx 0.02$ mag.

The light curves of both stars show a long time scale behaviour (several hundred days $\Delta V_{\mathrm{E}}=0.2-0.4 \mathrm{mag}$ ) with superimposed short time scale irregular variability (several days, $\Delta V_{\mathrm{E}}=0.1 \mathrm{mag}$ ). They both have a bluer when fainter behaviour for the long time-scale variation and the short time-scale variation. The very irregular photometric behaviour on short time scales is characteristic of Herbig AeBe stars, but may also be compatible with classical Be stars. We will come back to this in Sect. 5 .

\subsection{The photometry}

We noted that ESHC1 is catalogued by Meysonnier \& Azzopardi (1993) as an emission line star in their objective prism survey, and identified as Lin 232 in the Simbad database. Despite the fact that ESHC2 was in the field observed by Meysonnier \& Azzopardi, it was not detected as an $\mathrm{H} \alpha$ emitting object.

On January 7, 1998 (JD 2450821.39) we obtained $B V R I \mathrm{H} \alpha \mathrm{H} \alpha_{\text {off }}$ photometry at ESO La Silla with DFOSC on the Danish $1.5 \mathrm{~m}$ for the SMC field, with exposure times of $60 \mathrm{~s}, 30 \mathrm{~s}, 30 \mathrm{~s}, 30 \mathrm{~s}, 600 \mathrm{~s}$ and $600 \mathrm{~s}$ respectively. The filters are respectively numbered ESO 450, 451, 452, $425,693,697$. The CCD is a LORAL $2048 \times 2048$ CCD with a pixel scale of 0.39 arcsec. The data were reduced using QUYLLURWASI, the pipeline developed around 
Table 1. Photometry obtained for ESHC1 and ESHC2 with DFOSC on the Danish telescope at ESO La Silla on January 7, 1998 (indicated by a "d") and from the published catalogue of average SMC BVI photometry by OGLE (indicated by a "og") .

\begin{tabular}{llrrrrlrr}
\hline Name & $V \mathrm{~d}$ & $(B-V) \mathrm{d}$ & $(V-R) \mathrm{d}$ & $(V-I) \mathrm{d}$ & $\mathrm{H} \alpha-\mathrm{H} \alpha_{\text {off }}$ & $V$ og & $(B-V) \mathrm{og}$ & $(V-I) \mathrm{og}$ \\
\hline ESHC1 & $15.09 \pm 0.02$ & $0.03 \pm 0.02$ & $0.06 \pm 0.02$ & $0.11 \pm 0.02$ & $-0.42 \pm 0.03$ & $15.07 \pm 0.01$ & $-0.00 \pm 0.03$ & $0.13 \pm 0.04$ \\
ESHC2 & $17.03 \pm 0.02$ & $-0.03 \pm 0.02$ & $0.05 \pm 0.02$ & $0.10 \pm 0.02$ & $-0.58 \pm 0.03$ & $17.01 \pm 0.02$ & $-0.05 \pm 0.03$ & $0.07 \pm 0.04$ \\
\hline
\end{tabular}
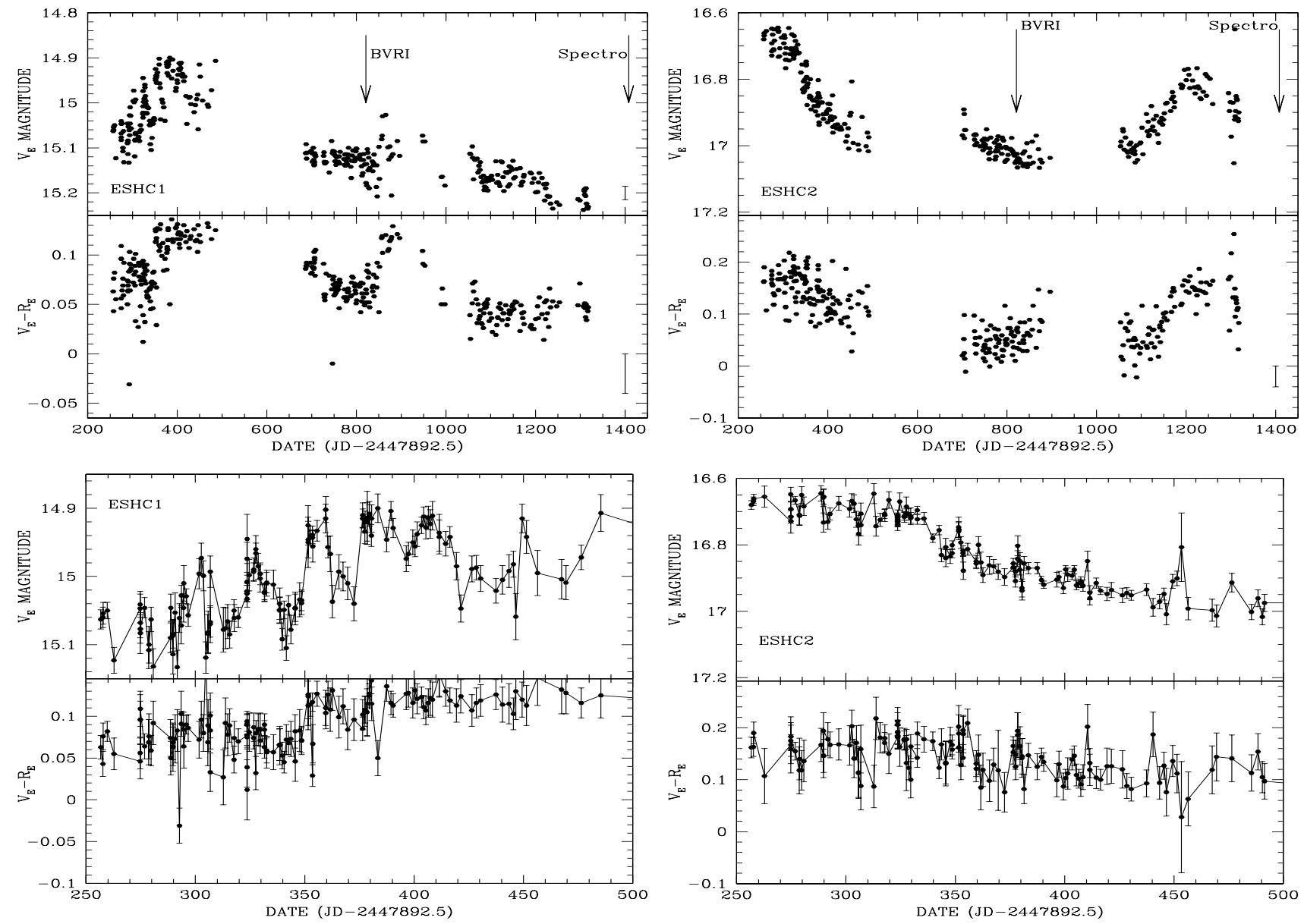

Fig. 2. Light curves of the two targets. The two upper panels show the full $V_{\mathrm{E}}$ and $V_{\mathrm{E}}-R_{\mathrm{E}}$ light curves for ESHC1 and ESHC2. The two lower panels show zooms on a part of the light curves. Notice the low amplitude irregular photometric variability. The time is given as JD-2447892.5. A typical error bar is given in the lower right of the two upper panels.

DoPhot for the PLANET collaboration. The absolute calibration was done using Landolt standards taken during the observations, leading to absolute calibration to $2 \%$ for stars brighter than $V=17 \mathrm{mag}$, and of the order of $5 \%$ for stars of $V=18 \mathrm{mag}$.

Finally, using WCSTOOLS and the MACS catalogue (Tucholke et al. 1996), we performed astrometry of the reference frames and the subtracted frames. Comparison with other catalogues indicates that we have an accuracy better than 0.5 arcsec.

We also searched for the program stars in the publicly available OGLE $B V I$ catalogues for the SMC of 2.2 million stars. These magnitudes are mean magnitudes over the observing period covered by OGLE (http://www.astrouw.edu.pl/ ftp/ogle/). The photometry is summarized in Table 1.
Moreover, narrow-band imaging in $\mathrm{H} \alpha, \mathrm{H} \alpha$ off was obtained with EFOSC2 mounted on the ESO $3.6 \mathrm{~m}$ at La Silla on August 18, 1999 (Julian day 2451408.79) for the field containing the targets with exposure times of 600 s. Flatfielding, bias subtraction and cosmic subtraction were performed with MIDAS $98 \mathrm{NOV}$ in the standard way. Although the seeing was poor (1.3 arcsec), these images are useful to search for $\mathrm{H} \alpha$ emitters in the field. We built subtracted images ( $\left.\mathrm{H} \alpha-\mathrm{H} \alpha_{\text {off }}\right)$ using ISIS, the new image subtraction package with a non-constant kernel (Alard 2000). The procedure is the following: we choose the $\mathrm{H} \alpha$ image as being the template. We re-map the $\mathrm{H} \alpha$ off image by bicubic spline interpolation from the $\mathrm{H} \alpha$ image. Then we adjust the parameters of ISIS to our images, and do several tests with different degrees to fit the variation of the convolution kernel over the image. We also performed 


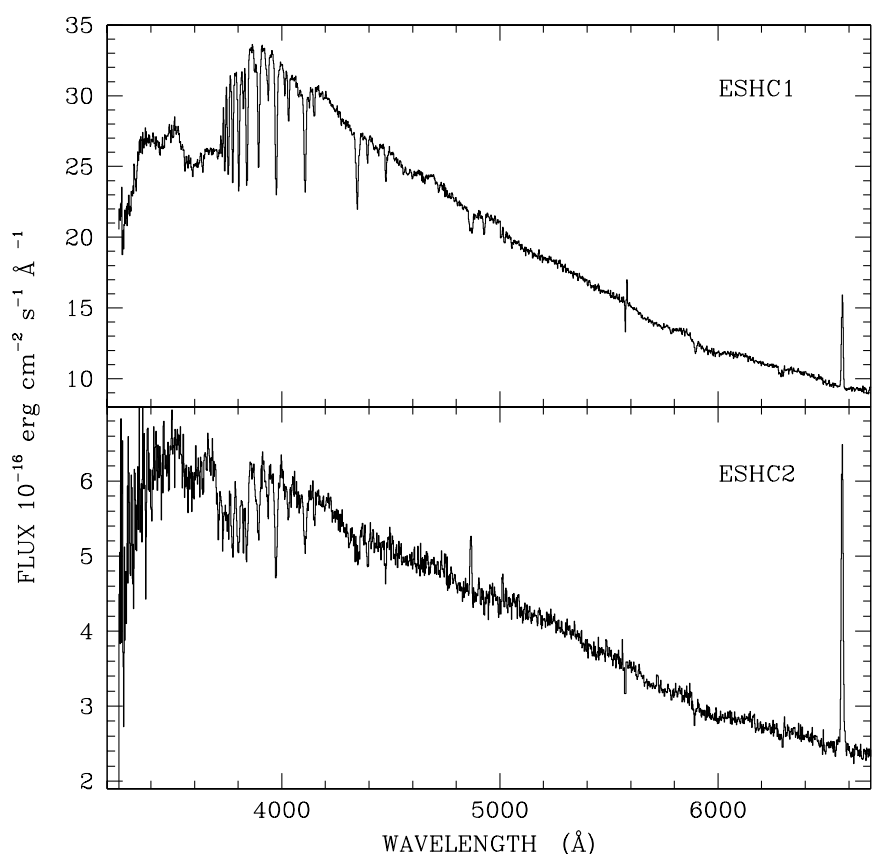

Fig. 3. Full spectrum of the two targets taken with EFOSC2 on the ESO $3.6 \mathrm{~m}$. The spectra have a resolution of $6 \AA$.

several tests to fit the background variations. The best results are obtained with a degree 1 variation in both the kernel and the background.

We present images of the $\left(\mathrm{H} \alpha-\mathrm{H} \alpha_{\text {off }}\right) / \sqrt{\mathrm{H} \alpha}$ flux in the lower left panel and the right panel of Fig. 1. It gives an idea of the noise statistics compared to the photon noise: the gray scale has been adjusted so that black means 4 sigma above photon noise level. It is clear that both ESHC objects are strong $\mathrm{H} \alpha$ emitters. They lie on a faint HII region illuminated by nearby hot stars. Notice that at $5 \operatorname{arcsec}(1.5 \mathrm{pc}$ on the sky) to the North West of ESHC2, there is another $\mathrm{H} \alpha$ emitter. Moreover, there are several $\mathrm{H} \alpha$ emitters clustered in the upper left corner of the field. This indicates that we are most likely in an area where star formation was active recently.

\subsection{The spectroscopic observations}

Spectroscopic observations were obtained with EFOSC2 mounted on the ESO $3.6 \mathrm{~m}$ at La Silla on August 18, 1999. The gratings Nos. 7 and 9 were used to cover the full spectral range from 3200 to $7000 \AA$. We used a $1.2 \operatorname{arcsec}$ slit, and we binned the data to a resolution of $6 \AA$. Multiple exposures of the two stars were made with the grating No. 7 (1200 s exposure), and grating No. 9 (900 s exposure) for each star. The spectra were reduced in a standard way using MIDAS 98NOV release and the LONG context. A signal to noise ratio of 200 was obtained for ESHC1 and 50 for $\mathrm{ESHC} 2$ in the blue part of the spectrum. A somewhat lower signal to noise ratio was obtained in the red part of the spectrum: 100 for ESHC1 and 30 for ESHC2.

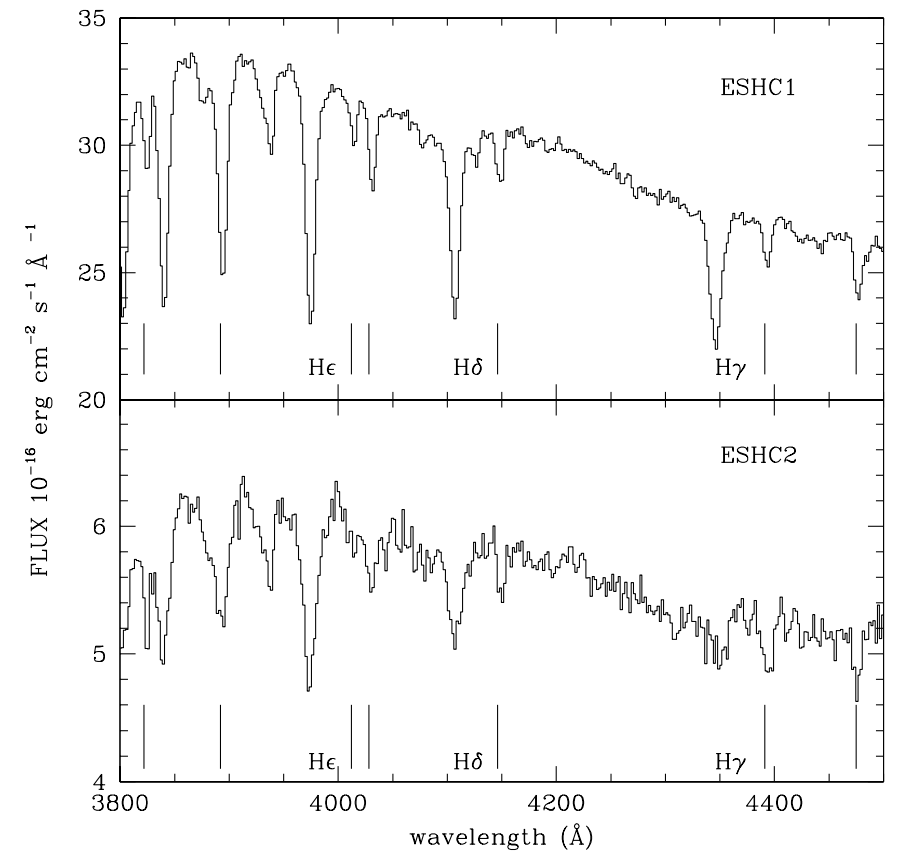

Fig. 4. The blue part of the spectrum of ESHC1 and ESHC2. Balmer lines $\mathrm{H} \epsilon, \mathrm{H} \delta, \mathrm{H} \gamma$ are labeled, and the solid lines vertical indicate HeI lines at 3822, 3892, 4012, 4026, 4146, 4387 and $4471 \AA$.

\section{Discussion of the spectra}

The full spectra are presented in Fig. 3 with ESHC1 in the upper panel and ESHC2 in the lower panel. The region between $3800<\lambda<4500 \AA$ is enlarged in Fig. 4 . The $\mathrm{H} \beta$ and $\mathrm{H} \alpha$ profiles are shown in Figs. 5 and 6 . We immediately notice that these two stars have stellar spectra with small Balmer jumps, clear Balmer lines in absorption and strong $\mathrm{H} \alpha$ emission. This suggests spectral type Be.

In order to improve the estimated spectral type, we decided to adopt the same approach as in LBD. We first looked for the presence or absence of classification lines, Balmer lines, HeI, HeII, CIII, OII, MgII, SiII, SiIV. When it was possible, we measured the position and equivalent width of the lines. It is clear that the metal lines are weak, as expected for metal-poor stars in the SMC, and marginally detected if detected at all. Therefore, in the following we will concentrate on the Balmer lines and the helium lines. The results are reported in Table 2. In the next two subsections we will discuss the spectral type of the two program stars.

\subsection{The spectrum of ESHC1}

ESHC1 shows $\mathrm{H} \alpha$ in emission, $\mathrm{H} \beta$ is partly filled with emission, all other Balmer lines from $\mathrm{H} \gamma$ to $\mathrm{H} 12$ and Helium I (3819, 3888, 4026, 4387, 4471, 4713, 4921, $5015,6678 \AA)$ are present, and clearly seen in absorption (Fig. 4). This star has a clear Balmer jump. The HeII line at $4686 \AA$ is absent. There may be a weak contribution of the CIII/OII blend at 4650, but if present, its equivalent width would be less than $0.1 \AA$. There may be a trace of 
Table 2. Lines identified in ESHC1 and ESHC2. We give the measured wavelength of the lines (Cols. 1, 5), the Full Width at Half Maximum (given in Cols. 2, 6), the equivalent width (Cols. 3, 7) and the measured Flux (in Cols. 4, 8).

\begin{tabular}{|c|c|c|c|c|c|c|c|c|}
\hline \multicolumn{4}{|c|}{ Star ESHC1 } & \multicolumn{4}{|c|}{ Star ESHC2 } & \multirow[b]{2}{*}{ Identification } \\
\hline $\begin{array}{c}\text { position } \\
(\AA)\end{array}$ & $\begin{array}{c}F W H M \\
(\AA) \\
\end{array}$ & $\begin{array}{l}E W \\
(\AA)\end{array}$ & Flux & $\begin{array}{c}\text { position } \\
(\AA)\end{array}$ & $\begin{array}{c}F W H M \\
(\AA)\end{array}$ & $\begin{array}{l}E W \\
(\AA) \\
\end{array}$ & Flux & \\
\hline 3822.8 & 6.0 & -0.54 & -17 & & & & & HeI 3819 \\
\hline 3892.0 & 10.4 & -3.2 & -106 & 3889.8 & 18.0 & -2.7 & -16 & HeI 3888 \\
\hline 4012.2 & 6. & -0.4 & -12 & & & & & HeI 4009 \\
\hline 4029.4 & 7. & -0.8 & -23 & 4028.4 & 11 & -0.8 & -4.5 & HeI 4026 \\
\hline 4146.5 & 7.6 & -0.6 & -17.5 & 4147.7 & 7 & -0.7 & -4 & HeI 4146 \\
\hline 4391.5 & 9.3 & -0.74 & -20.2 & 4392.0 & 7.6 & $-0.8-1.0$ & & HeI 4387 \\
\hline 4475.1 & 8.8 & -0.83 & -22 & 4473.8 & 6 & -0.6 & -3 & HeI 4471 \\
\hline 4717.3 & 7.5 & -0.28 & -6.8 & & & & & HeI 4713 \\
\hline 4925.7 & 6.8 & -0.62 & -13 & 4925.0 & 7.4 & -0.7 & -3.4 & HeI 4921 \\
\hline 5019.6 & 6.2 & -0.23 & -4.65 & & & & & HeI 5015 \\
\hline 6684.3 & 10.9 & -0.6 & -5.6 & & & & & HeI 6678 \\
\hline 5894.5 & 12.6 & -1.0 & -13 & 5890.6 & 14 & -1.0 & -3.2 & $\mathrm{Na}$ \\
\hline 3737.3 & 6.0 & -1.1 & -33 & & & & & $\mathrm{H}_{13} 3734$ \\
\hline 3753.0 & 7.5 & -1.7 & -51 & & & & & $\mathrm{H}_{12} 3750$ \\
\hline 3773.4 & 7.9 & -2.1 & -65 & 3773.1 & 13 & -2 & -11 & $\mathrm{H}_{11} 3770$ \\
\hline 3800.9 & 9.12 & -2.58 & -81 & 3800 & 14 & -2.3 & -13 & $\mathrm{H}_{10} 3798$ \\
\hline 3838.4 & 8.8 & -2.5 & -81 & & & & & HeI $3838+\mathrm{H}_{9} 3835$ \\
\hline 3936.8 & asym & & & 3934.2 & 9.7 & -0.9 & -5.4 & $\mathrm{Ca} \mathrm{H}$ \\
\hline 3972.7 & 10.4 & -3.4 & -110 & 3972.1 & 13.9 & -4.0 & -24 & $\mathrm{H} \epsilon / \mathrm{H} \epsilon+\mathrm{K}$ \\
\hline 4105.1 & 9.7 & -2.33 & -70 & 4105.4 & 14.1 & -2.7 & -16 & $\mathrm{H} \delta$ \\
\hline 4344.2 & 12.7 & -2.54 & -69 & & filled & & & $\mathrm{H} \gamma$ \\
\hline 4865.5 & & ly filles & & 4864.5 & 9 & 2 & 9 & $\mathrm{H} \beta$ \\
\hline 6567.8 & 7.5 & 6.2 & 58 & 6567.4 & 10.1 & 18.5 & 40.4 & $\mathrm{H} \alpha$ \\
\hline
\end{tabular}

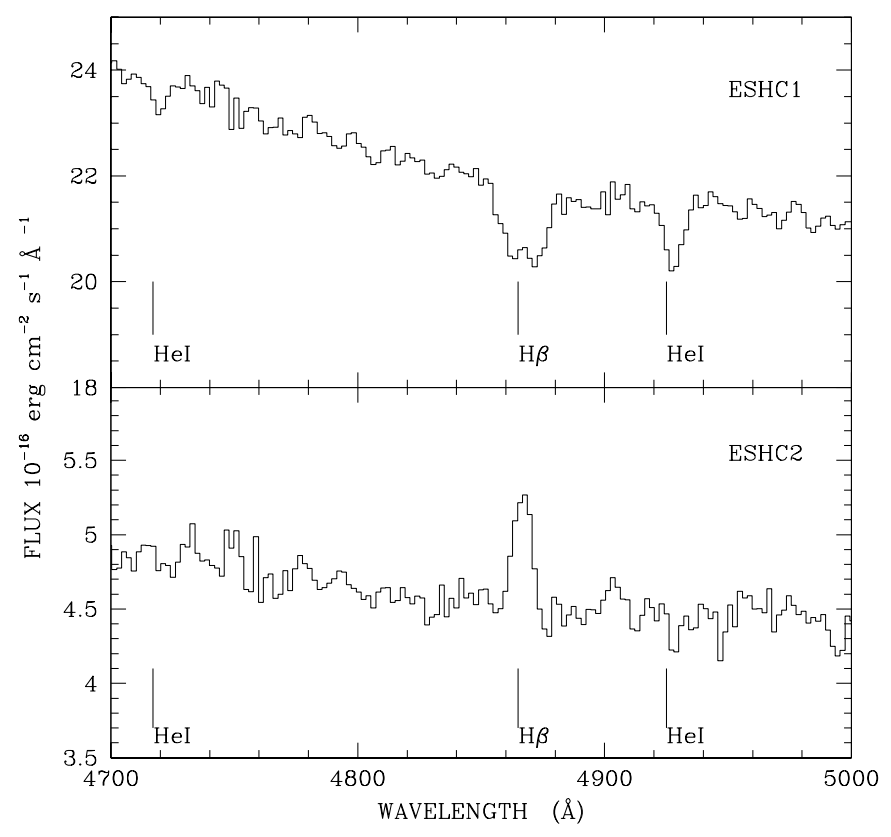

Fig. 5. The $\mathrm{H} \beta$ line and HeI lines at $4713 \AA$ and $4925 \AA$ of ESHC1 and ESHC2.

OII at $4662 \AA$. There may also be a trace of SiIV at 4116 , but the SiIV counterpart at $4089 \AA$ is in the wing of $\mathrm{H} \epsilon$. Therefore the presence of these lines is inconclusive. The MgII doublet at $4481 \AA$ is not detected.

We compare the data with the tables from Didelon (1982) of the equivalent width as a function of spectral

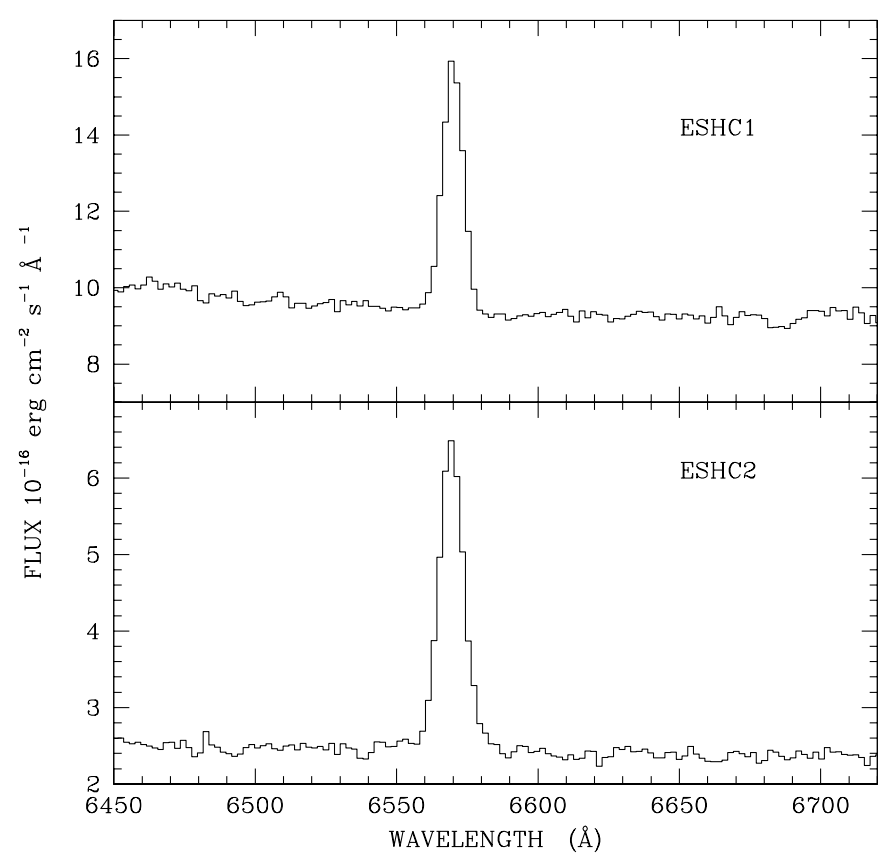

Fig. 6. The $\mathrm{H} \alpha$ line of ESHC1 and ESHC2.

type for several lines. Most of the metal lines do not give strong constraints in this particular case. However, the presence and strength of the $\mathrm{H}$ and HeI lines suggest that this star is a Be star with a luminosity class III, IV or V. The spectral type and luminosity class is best constrained by the HeI 4387 line, which indicates a luminosity class III, 
Table 3. Summary of stellar parameters for ESHC1 and ESHC2.

\begin{tabular}{llllc}
\hline Name & Type & $\log T_{\text {eff }}$ & $\log \left(L / L_{\odot}\right)$ & $\left(R / R_{\odot}\right)$ \\
\hline ESHC1 & B4 III & $4.20 \pm 0.02$ & $4.23 \pm 0.06$ & $17.4 \pm 1.2$ \\
ESHC2 & B2 IV-V & $4.34 \pm 0.06$ & $3.84 \pm 0.06$ & $6.1 \pm 1$ \\
\hline
\end{tabular}

and a spectral type B4-6. The HeI 4471 line also implies that the star has a type between B4 and B6, but HeI 4026 line implies a slightly earlier spectral range of B1 to B4. Based on these lines, we classify this star as a B4e III.

\subsection{The spectrum of ESHC2}

ESHC2 shows $\mathrm{H} \alpha$ and $\mathrm{H} \beta$ in emission. All other Balmer lines, from $\mathrm{H} \gamma$ to $\mathrm{H} 13$, and $\mathrm{HeI}(3888,4026,4143,4392$, $4471 \AA$ ) are present and clearly in absorption. This star has a weaker Balmer Jump than ESHC1. The HeII line at $4686 \AA$ and the MgII doublet at $4481 \AA$ are absent. No features are seen at $4650 \AA$ from the CIII/OII blend. As for ESHC1, we used the tables of Didelon (1982) on the HeI lines at 4471, 4026, 4387 and $4120 \AA$ to determine the spectral type and luminosity. Based on the comparison of the observed line strength with Didelon's tables we classify this star as a B2e IV-V.

\subsection{Spectral type from the $Q$ method}

We can also estimate the spectral type from the photometry, using the relation between the reddening-free $Q$-parameter and the spectral type. For this purpose we adopt the transmission curves of the $U, B$ and $V$ filters and convolve these with the observed spectra to derive the $U$, $B$ and $V$ magnitude in the Johnson system. The resulting colours are for ESHC1 $(U-B=-0.45, B-V=0.04)$ and for ESHC2 $(U-B=-0.62$ and $B-V=0.20)$.

We apply the seminal Q method (Johnson \& Morgan $1953)$ to determine reddening-free colour $Q=(U-B)-$ $0.72(B-V)$. We derived $Q=-0.47$ for ESHC1 and $Q=-0.76$ for ESHC2. Using the relation between $Q$ and spectral type for Be stars by Halbedel (1993) we derive a spectral type B5 and B2 respectively. This agrees very well with the spectral types of B4eIII and B2eIV-V derived from the $\mathrm{H}$ and HeI lines above.

A word of caution may be necessary here. The $Q$ versus spectral type relation that we adopted is derived for Galactic stars. The metal-poor SMC stars, which have less line blanketing and line blocking may have different colours than Galactic stars of the same $T_{\text {eff }}$, and therefore different temperatures than the Galactic stars of the same $Q$-value. However, comparing the colours and magnitudes of the Kurucz model atmospheres (1979) for $Z=0.02$ (Galactic) and $Z=0.002$ (lower than SMC), we find that the difference is very small. Given the uncertainty in the method and the fact that the $\mathrm{H}$ and $\mathrm{HeI}$ lines indicate the same type as the $Q$-method, we will adopt these spectral types and their corresponding values of $T_{\text {eff }}$ from Galactic stars.

\section{Determination of the stellar parameters $T_{\text {eff }}$, $L, M, R$}

\section{1. $E S H C 1$}

We adopt a spectral type B4 III for ESHC1 as the most reasonable estimate. From the OGLE photometry the mean magnitude and colours are $V=15.08 \pm 0.01$ and $(B-V)=0.00 \pm 0.03$. The intrinsic colour of a B4III star is $(B-V)_{0}=-0.19$ (Lang 1991), which results in $E(B-V)=0.19 \pm 0.03$. We make the assumption that the colour difference is due to obscuration by normal dust with $R_{V}=3.3$ (Cardelli et al. 1989). This results in an intrinsic magnitude of $V_{0}=14.45 \pm 0.11$. Adopting a distance modulus of $18.94 \pm 0.04$ (Laney \& Stobie 1994) to the SMC, we find $M_{V}=-4.49 \pm 0.15$. With a bolometric correction of -1.45 for spectral type B4III (Lang 1991) this yields $M_{\text {bol }}=-5.94 \pm 0.15$ or $\log \left(L / L_{\odot}\right)=4.23 \pm 0.06$. The effective temperature of a B4III star is $\log T_{\text {eff }}=4.20 \pm 0.02$ (Lang 1991) and so $\log \left(R / R_{\odot}\right)=1.24 \pm 0.03$ and $R=17.4 \pm 1.2 R_{\odot}$. Following Lang, we use an empirical Mass Luminosity relation $\log \left(M / M_{\odot}\right)=0.48-0.105 M_{\text {bol }}$ and derive a mass of $M \approx 13 M_{\odot}$. Similar results are obtained when we derive the parameters with the photometry we obtained from ESO telescope.

\section{2. $E S H C 2$}

We adopt a spectral type B2 IV-V for ESHC2 as the most reasonable estimate. From the OGLE photometry the mean magnitude and colours are $V=17.01 \pm 0.02$ and $(B-V)=-0.06 \pm 0.03$. Adopting the intrinsic colour $(B-V)_{0}=-0.24$, an effective temperature of $\log T_{\text {eff }}=4.34 \pm 0.02$ and a bolometric correction of $B C=-2.35$ from Lang (1991) we find the following stellar parameters: $V_{0}=16.42 \pm 0.12, M_{V}=-2.52 \pm 0.16$, $M_{\text {bol }}=-4.87 \pm 0.16, \log \left(L / L_{\odot}\right)=3.84 \pm 0.06$ and $R=6.1 \pm 1 R_{\odot}$. We get a mass of $M \approx 10 M_{\odot}$. Again, similar results are obtained when we derive the parameters with the photometry we obtained from the ESO telescope. The results are summarized in Table 3.

\section{Discussion}

We have presented two stars with the following characteristics:

(1) early B spectral type with strong $H \alpha$ emission;

(2) irregular photometric behaviour with time scales of 20-200 days;

(3) a bluer photometric colour, during brightness minimum;

(4) located within a projected distance of $20 \mathrm{pc}$ of each other in a weak HII region;

(5) positioned in the HR-diagram to the right of the Main Sequence (see below).

The five points suggest that the stars might be Herbig AeBe or classical Be stars. If they are PMS stars, then 
they are the first discovered Pre-Main Sequence stars in the SMC (see also Hutchings \& Thompson 1988).

We rule out that the objects are $\mathrm{B}[\mathrm{e}]$ supergiants, as no forbidden line emission was detected. Moreover the ESHCs have a lower luminosity class, at variance with the supergiant nature of B[e] stars in the Magellanic Clouds (Lamers et al. 1998).

\subsection{Comparing the properties of ESHC1 and ESHC2 with classical Be and $\mathrm{HAeBe}$ stars}

To make a distinction based on visual properties between the two possible classifications is precarious as many properties are common to both classical Be stars and Herbig Be stars. However we might find some indications, by having a closer look at the characteristics mentioned above. The known physical processes (i.e. bound-free and freefree continuum emission, denoted bf and ff respectively hereafter) which occur in the CS environment of classical Be stars allow predictions with respect to the $H \alpha$ emission and colour excess $E(B-V)$. In contrast, the activity exhibited by Galactic HAeBe stars continues to fuell active debate, and therefore no quantitative predictions can be made.

- The $\mathbf{H} \boldsymbol{\alpha}$ emission of Classical Be stars is correlated with the observed colour excess (Dachs 1988). It is expected that a larger contribution of the bf-ff excess emission will generate larger $H \alpha$ emission accompanied by a larger colour excess. Early type Galactic classical Be stars have a maximal colour excess of $E(B-V) \sim 0.10$ (Waters et al. 1987). The derived $E(B-V)$ for ESHC1 and ESHC2 are 0.19 and 0.18 . If we correct for the average extinction towards the SMC $(E(B-V)=0.09$, Massey et al. 1995), we find that the colour excesses of ESHC1 and ESHC2 are similar to the maximum observed for the early type Galactic Be stars (Waters et al. 1987). However, comparing the colour excess with the $H \alpha$ emission for classical Be stars done by Dachs (1988) we noticed that the colour excess is much larger than expected on the basis of the $H \alpha$ emission. This might be attributed to additional circumstellar extinction. In general Galactic HAeBe have colour excesses which exceed the level observed for classical Be stars.

- The irregular brightness variability of Classical Be stars has been shown to be largest among spectral types B1-B2, on the basis of the four years (similar to the EROS2 data) of observations of Hipparcos (Hubert \& Floquet 1998). Amplitudes of variability in the Hipparcos passband were measured to have maximum values of $\Delta H p=0.3$, associated with outbursts on time spans of hundreds of days. This variability is accompanied by small amplitude $\left(<0.1^{\mathrm{m}}\right)$ variation on time scales of days, often periodic (e.g. Pavlovski et al. 1997; Hubert \& Floquet 1998). The amplitude of the variability of the ESHCs is slightly larger the classical Be stars, however the Hipparcos filter is wider and extends further to the blue part of the spectrum than the EROS2 $V_{E}$ passband.
Bf-ff emission has its largest contribution in the red part. Therefore the amplitude in the EROS2 passband is expected to be slightly larger than the Hipparcos passband. The superposed short time scale variability of the ESHCs ( $\sim 20$ days $)$, is however not reported for Be stars by Hubert \& Floquet (1998). This time-scale of variability is however observed among the HAeBe stars (Herbst \& Shevchenko 1999).

- The observed colour variability of the ESHCs can be interpreted in terms of physical processes, which are thought to occur in the CS environment of classical Be stars (Dachs 1982; Waters et al. 1987). However this explanation is not unique. Just like the two SMC HAeBe candidates, most of the LMC Pre-Main Sequence candidates also show bluer-when-fainter behaviour. In LBD we discuss the possibility of explaining the observed photometric and colour variability in terms of dust obscuration and the existence of a reflection nebula. The existence of a reflection nebula is one of the original selection criteria of Herbig (1960). The total flux of HAeBe stars at large distances (like in the LMC and in the SMC), will be contaminated by the unresolved, blue reflection nebula (typical sizes of Galactic reflection nebulae, $\sim 0.25 \mathrm{pc}$, will be less than 1 arcsec in the Magellanic Clouds). The nebula will also cause the colour excess to be small. In LBD it was concluded that one would preferentially observe Magellanic Cloud HAeBes having a bluer-when-fainter colour behaviour due to dust obscuration.

- The close proximity of the two ESHCs to each other would mildly support the HAeBe classification. However, we cannot exclude the chance nearby projection of two irregularly variable classical Be stars. The H II region does indicate the presence of hot stars and thus a region which might have recently witnessed star formation.

\subsection{Position in the HR-diagram}

We have seen in the previous subsection that the (visual) evidence does not point towards one particular classification (classical Be or Herbig Be) of the ESHCs. In the two panels of Fig. 7 we present an HR-diagram. The purpose is to compare the derived stellar parameters of the ESHCs with the Classical Be stars and the Herbig Ae/Be stars. The ESHCs are presented as filled symbols. The Galactic HAeBes are plotted as crosses, and the open symbols are their counterpart candidates in the LMC, the ELHCs. The full line is the Main-Sequence.

The dotted lines in the upper panel of Fig. 7 represent the "birthline" for pre-Main Sequence stars for two different proto-stellar mass accretion rates. The lower dotted line is the canonical Galactic birthline for a accretion rate of $10^{-5} M_{\odot} \mathrm{yr}^{-1}$, and higher dotted line is the birthline for an accretion rate $10^{-4} M_{\odot} \mathrm{yr}^{-1}$ (Palla \& Stahler 1993). The crosses are Galactic HAeBe stars adopted from Berrilli et al. (1992). The observed upper limit for Galactic HAeBes agrees approximately with an accretion rate of $10^{-5} M_{\odot} \mathrm{yr}^{-1}$. We see, as noticed in $\mathrm{BL}$ and 

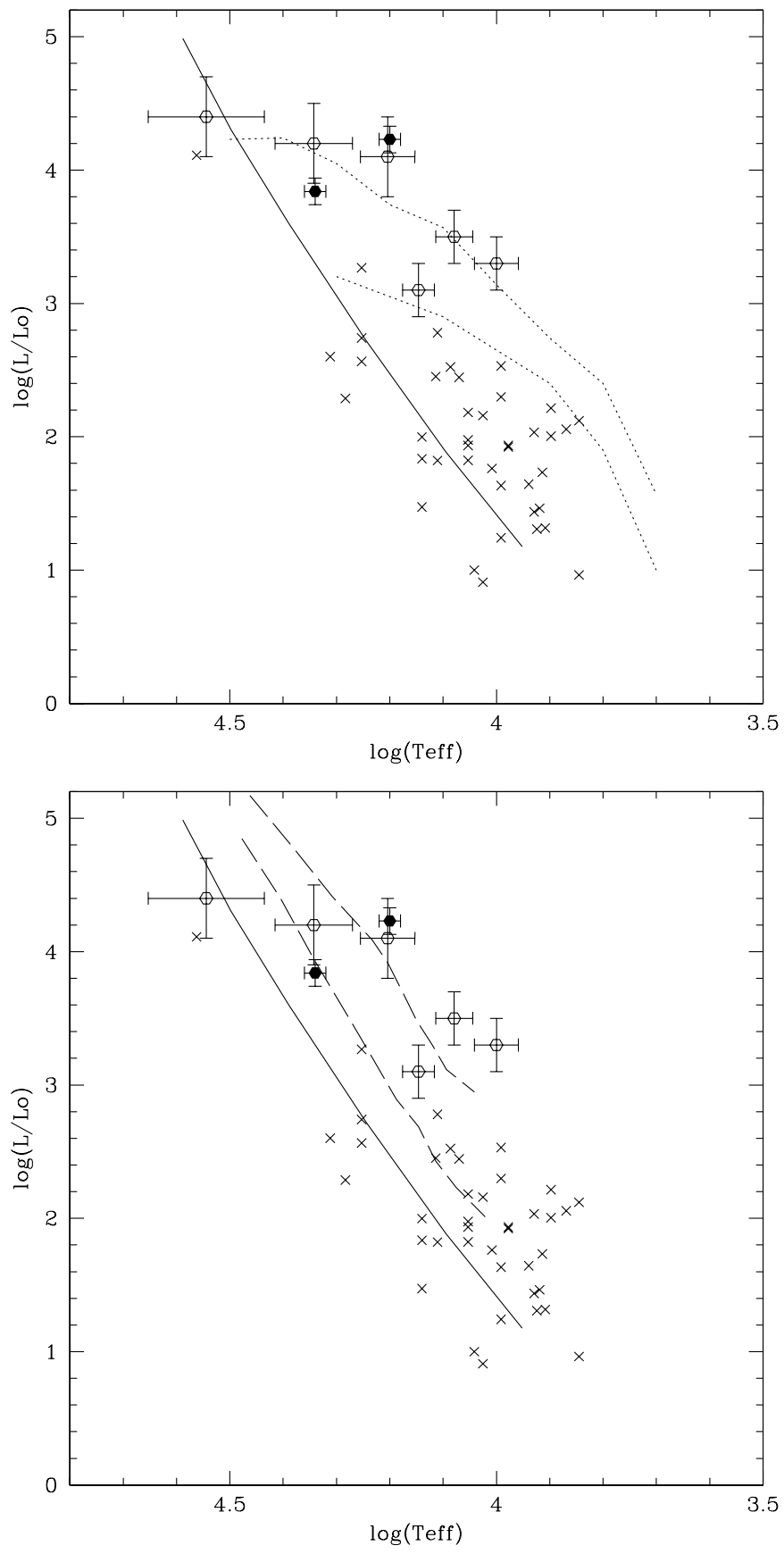

Fig. 7. Both panels are HR diagrams for Galactic (crosses, Berrilli et al. 1992), LMC (open circles, Lamers et al. 1999) Herbig Ae Be candidates and the two targets of this study. The main sequence is indicated as a line. The upper panel shows also the two birthlines from Palla \& Stahler (1993) for mass accretion rates of $10^{-5} M_{\odot} \mathrm{yr}^{-1}$ (long dashed lines) and $10^{-4} M_{\odot} \mathrm{yr}^{-1}$ (short dashed lines). The lower panel shows (long dashed lines) the upper limit for main sequence Be stars and giant Be stars, adopted from Zorec \& Briot (1997).

LBD, that some of the LMC HAeBe candidates (open symbols) are more luminous by a factor of 3 to 10 than the Galactic birthline. In comparison the luminosity of SMC star ESHC1 complies with the high luminosity found for the LMC HAeBe candidates.
The luminosity of the birthline depends on the accretion rate. We found that some of the ELHCs and ESHC1 agree better with the birthline corresponding to a fast accretion rate of $10^{-4} M_{\odot} \mathrm{yr}^{-1}$. Just based on these two stars, we cannot compare birthlines in LMC and SMC yet, but it is clear that these two pre-main sequence candidates in the SMC are above the Galactic birthline, and are very similar to the LMC pre-main sequence candidates. If indeed they are pre-main sequence stars, it would support our previous conclusion that, at low metallicity, we can observe more massive stars in pre-main sequence phases than in our Galaxy.

Palla (2000, private communication) has argued that massive Galactic PMS may also be above the birthline for an accretion rate of $10^{-5} M_{\odot} \mathrm{yr}^{-1}$. If that is the case, the high location of ELHCs and ESHCs in the HRD may not be necessarily related to the low metallicity. On the other hand the massive PMS stars noted by Palla are closer to the Main Sequence than the ELHCs and ESHCs. So, in the temperature range $10000<T_{\text {eff }}<15000$, the LMC and $\mathrm{SMC} \mathrm{HAeBe}$ candidates are definitively above the Galactic HAeBe stars.

On the other hand, we consider the case that they can be classical Be stars. The dashed lines in the lower panel of Fig. 7 indicate the upper limit for main sequence and giant classical Be stars. These two upper limits have been derived from Zorec \& Briot (1997). In this work the average $V$-Magnitude excess of classical Be stars has been derived as function of B spectral subtype. We added this excess to the the absolute visual magnitude (also given in Zorec \& Briot 1997), in which we included the dispersion $\sigma_{M_{V}}$ in order to obtain the upper limit. The effective temperature and bolometric correction were adopted from SchmidtKaler (1982). The figure shows that the stellar parameters of ESHC2 are compatible with the derived classical Be upper limits for main sequence stars, and ESHC1 with the upper limits for giants.

It is not possible to unambiguously distinguish between $\mathrm{HAeBe}$ and classical Be interpretation for the two stars!

\section{Conclusions}

We have identified two blue irregular variables in the SMC with spectral type B4IIIe (ESHC1) and B2IV-Ve (ESHC2), with strong $H \alpha$ emission. These stars could be either classical Be stars or pre-main Sequence HAeBe stars. We find evidence for both interpretations:

- The H $\alpha$ emission of the ESHCs stars is much larger than that of the classical Be stars with the same colour excess.

- The short timescale variability of the ESHC stars of about 20 days is similar to that of HAeBe stars, but not found in classical Be stars.

- The bluer-when-fainter colour variability could be explained by variable free-free and bound-free emission (as in classical Be stars) or by variable extinction of a 
star with a blue scattering nebula that is not resolved (as expected for HAeBe stars at the SMC distance).

- The presence of a nearby H II region and the proximity of clusters of $\mathrm{H} \alpha$-emitters indicates recent star formation, which would favour the HAeBe interpretation.

So we find a mild preference for the interpretation of the ESHC stars as HAeBe stars. On the other hand, the possibility that the stars are classical Be stars with abnormal photometric properies cannot be excluded. Infrared observations and high resolution observations are needed to confirm or deny one of the two possibilities, because HAeBe stars are known to have an IR excess due to dust emission and HAeBe stars are often surrounden by a reflection nebula.

If the stars turn out to be HAeBe stars indeed, they are the first pre-main sequence stars found in the SMC. Their magnitude then indicates that they are located above the Galactic birthline in the HR-diagram, which suggests a faster accretion rate than their Galactic counterparts.

Acknowledgements. This work is based on data obtained at ESO La Silla. We thank Dr. Zorec for useful discussions and Dr. Grinin for constructive comments on the manuscript. S.T. thanks Dr. Belial for methodological approach. This work was partly supported by the Van Gogh program. We are grateful to D. Lacroix and the technical staff at the Observatoire de Haute Provence and to A. Baranne for their help in refurbishing the MARLY telescope and remounting it in La Silla. We are also grateful for the support given to our project by the technical staff at ESO, La Silla. We thank J. F. Lecointe for assistance with the online computing.

\section{References}

Alard, C. 2000, in The Impact of Large-Scale Surveys on Pulsating Star Research, ASP Conf. Ser., 203, IAU Colloq., 176,50

Beaulieu, J. P., Lamers, H. J. G. L. M., Grison, P., et al. 1996, Science, 272, 995

Berrilli, F., Corciulo, G., Ingrosso, G., et al. 1992, ApJ, 398, 254
Bibo, E. A., \& Thé, P. S. 1991, A\&AS, 89, 319

Cardelli, J. A., Clayton, G. C., \& Mathis, J. S. 1989, ApJ, 345, 245

Dachs, J. 1982, in Be Stars, IAU Symp., 98, 19

de Wit, W. J. M, Beaulieu, J. P., \& Lamers, H. J. L. M. 2001a, A\&A, submitted

de Wit, W. J. M, Beaulieu, J. P., Lamers, H. J. L. M., Marquette, J. B., \& Lesquoy, E. 2001b, A\&A, submitted

Didelon, P. 1982, A\&AS, 50, 199

Finkezeller, U., \& Mundt, R. 1984, A\&AS, 55, 109

Halbedel, E. M. 1973, PASP, 105, 465

Herbig, G. H. 1960, ApJS, 4, 337

Herbig, G. H. 1994, in The Nature and evolutionnary status of Herbig Ae/Be stars, ed. P. S. Thé, M. R. Perez, \& E. P. J. van den Heuvel, ASP Conf. Ser., 62, 3

Herbst, W., \& Shevchenko, V. S. 1999, AJ, 118, 1043

Hubert, A. M., \& Floquet, M. 1998, A\&A, 335, 565

Hutchings, J. B., \& Thompson, I. B. 1988, ApJ, 331, 294

Johnson, H. L., \& Morgan, W. W. 1953, ApJ, 117, 113

Kurucz, R. L. 1979, ApJS, 40, 1

Lamers, H. J. G. L. M., Beaulieu, J. P., \& de Wit, W. J. 1999, A\&A, 341, 827

Lamers, H. J. G. L. M., Zickgraf, F.-J., de Winter, D., Houziaux, L., \& Zorec, J. 1998, A\&A, 340, L117

Laney, D., \& Stobie, R. 1994, MNRAS, 266, 441

Lang, K. R. 1991, Astrophysical data: Planets and stars (Springer-Verlag Berlin), 114

Massey, P., Lang, C. C., Degioia-Eastwood, K., \& Garmany, C. D. 1995, ApJ, 438, 188

Meysonnier, N., \& Azzopardi, M. 1993, A\&AS, 102, 451

Palla, F., \& Stahler, S. W. 1993, ApJ, 418, 414

Palanque-Delabrouille, N., Afonso, C., Albert, J. N., et al. (EROS Collaboration) 1998, A\&A, 332, 1

Schmidt-Kaler, T. 1982, in Landolt Bornstein, vol. 2, Subvol. B (Springer Verlag, Berlin)

Thé, S. 1994, in The Nature and evolutionnary status of Herbig Ae/Be stars, ed. P. S., Thé, M. R., Perez, \& E. P. J., van den Heuvel, ASP Conf. Ser., 62, 23

Waters, L. B. F. M., \& Waelkens, C. 1998, ARAA, 36, 233

Waters, L. B. F. M., Cote, J., \& Lamers, H. J. G. L. M. 1987, A\&A, 185, 206

Zorec, J., \& Briot, D. 1997, A\&A, 318, 443 